\section{A FORMAÇÃO EM SAÚDE COLETIVA NOS CURRÍCULOS DE EDUCAÇÃO FÍSICA: UM RETRATO ATUAL}

\author{
PUBLIC HEALTH TRAINING IN PHYSICAL EDUCATION CURRICULA: A \\ CURRENT PORTRAIT C
}

\section{LA FORMACIÓN EN SALUD COLECTIVA EN LOS CURRÍCULOS DE EDUCACIÓN FÍSICA: UN RETRATO ACTUAL C P}

doi) https://doi.org/10.22456/1982-8918.113041

\section{Viviana Graziela de Almeida Vasconcelos Barboni* <vivianabarboni@yahoo.com.br>}

Yara Maria de Carvalho*<yaramc@usp.br>

Vagner Herculano de Souza** <vagner_souza@usp.br>

\footnotetext{
*Universidade de São Paulo. São Paulo, SP, Brasil.

**Universidade Estadual de Ciências da Saúde de Alagoas. Maceió, AL, Brasil.
}

\begin{abstract}
Resumo: Este estudo apresenta uma análise nacional da formação superior em Saúde Coletiva nos cursos de graduação em Educação Física de instituições de ensino superior públicas. Foi realizada uma análise documental das estruturas curriculares, ementas e programas de disciplinas ofertadas por essas universidades, avaliando os conteúdos, a inserção prática no Sistema Único de Saúde (SUS) e a carga horária em Saúde Coletiva. Ao todo, 173 currículos ativos e regulares de graduação de 87 instituições públicas foram analisados, e observaram-se defasagens na formação em saúde: quando obrigatórias, as disciplinas de Saúde Coletiva se apresentam como componentes isolados; predominam conteúdos ligados às ciências biológicas; apresentam limitada carga horária e falta inserção e diálogo com os serviços de saúde do SUS. Identificaram-se algumas propostas curriculares que avançam na formação em saúde, mas ainda de maneira muito tímida, especialmente frente à urgência que a nova realidade decorrente da pandemia de covid-19 exige dos cursos de saúde.
\end{abstract}

Palavras chave: Educação Física. Educação Superior. Saúde Pública. Sistema Único de Saúde.
Recebido em: 15 abr. 2021 Aprovado em: 27 set. 2021 Publicado em: 11 nov. 2021

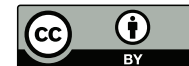

Este é um artigo publicado sob a licença Creative Commons Atribuição 4.0 Internacional (CC BY 4.0). elSSN: 1982-8918 


\section{INTRODUÇÃO ${ }^{1}$}

Educação Física é uma profissão de nível superior reconhecida como integrante multidisciplinar da equipe de saúde, estando como as demais e no núcleo de seu saber e competência habilitada para atuar na prevenção de doenças, promoção e recuperação da saúde (BRASIL, 1997).

As Diretrizes Curriculares Nacionais (DCN) para graduação em Educação Física direcionam a formação em duas vertentes: uma, para atuação na educação básica no curso de formação de professores (licenciatura) e outra, para o bacharelado, possibilitando que o profissional atue em diferentes cenários - saúde, esporte e lazer (BRASIL, 2018a).

Dessa forma, o campo denominado Saúde Coletiva com seus conteúdos apresenta aspectos importantes na formação em ambas as áreas da Educação Física. Enquanto os bacharéis se direcionam para o cuidado em saúde no Sistema Único de Saúde (SUS), na assistência ou na gestão, os licenciados se direcionam ao contexto escolar, de modo que os conteúdos da saúde estão inseridos nos currículos de Educação Física conforme previsto nos Parâmetros Curriculares Nacionais (BRASIL, 1998) e a Base Nacional Curricular Comum (BRASIL, 2018b).

Entretanto, a defasagem dos conteúdos da Saúde Coletiva nos currículos de graduação em Educação Física é amplamente conhecida em ambas as modalidades (LOCH; RECH; COSTA, 2020), ainda que o Conselho Nacional de Saúde (CNS), em 1997 (BRASIL, 1997), tenha reconhecido a área específica como subárea da saúde. A prevalência de perspectivas e conteúdos voltados para o fitness, treinamento e competição com forte viés das ciências naturais é também perceptível no âmbito da pós-graduação da área (MANOEL; CARVALHO, 2011).

No entanto, com a ampliação e consolidação do SUS e a abertura do campo de atuação conquistado pela Educação Física e outras subáreas da saúde (Fisioterapia, Farmácia, Terapia Ocupacional, Psicologia, entre outras), esse contexto vem se reorientando paulatinamente e exigindo uma importante readequação na formação de modo a possibilitar a atuação na saúde em acordo com os princípios e diretrizes das políticas públicas de saúde.

Ainda assim, diversos estudos têm apontado as enormes lacunas curriculares quanto à formação em saúde nos cursos de Educação Física em todo o país, tornando presente a reflexão sobre a necessidade de engajamento no processo de reformulação dos currículos em alinhamento com as questões voltadas para Saúde Coletiva (BRUGNEROTTO; SIMÕES, 2009; COSTA et al., 2012; OLIVEIRA; ANDRADE, 2016; OLIVEIRA; GOMES, 2020; PASQUIM, 2010).

A grande complexidade do sistema de saúde brasileiro exige dos profissionais da área conhecimentos, vivências e aprofundamentos nos diferentes cenários do SUS, para além dos saberes tradicionalmente contemplados na formação em Educação Física (controle motor, fisiologia do exercício, biomecânica e medidas antropométricas), considerados, dentro dos princípios do sistema de saúde, insuficientes para dar conta de uma atuação que atenda às necessidades sociais (BRASIL, 2018c).

1 O trabalho é produto da dissertação de mestrado em fase final da primeira autora. 
As DCN para os cursos de graduação em Educação Física reconhecem a necessidade de reorientar a formação, incluindo a saúde como um dos três eixos norteadores, além de esporte, cultura e lazer (BRASIL, 2018a). Esse marco, que ainda é recente, estabelece um importante momento de mudança que tem sido propulsor de reflexões, discussões e proposições de natureza acadêmico-científica e filosófica acerca da formação em saúde, de modo geral.

Nesse sentido, o que apresentamos neste artigo é decorrência de um estudo que vem realizando uma análise nacional da formação em Educação Física em Instituições de Ensino Superior (IES) públicas com intuito de melhor descrever como ocorre a interface com a Saúde Coletiva, a fim de também contribuir para com as discussões curriculares, reconhecendo que através dos currículos estabelece-se um jogo de forças, interesses, perspectivas que têm contribuído também para com o debate da identidade profissional.

\section{METODOLOGIA}

Trata-se de um estudo descritivo a partir de análise documental a respeito dos conteúdos da Saúde Coletiva nos cursos de graduação em Educação Física de universidades públicas federais e estaduais no Brasil. Foram avaliadas as estruturas curriculares da graduação, as ementas e os programas das disciplinas ofertadas. $O$ levantamento dos currículos levou em conta cursos cadastrados como "Educação Física" ativos no país, utilizando como base de busca a lista de cursos superiores de graduação e sequenciais expedida em abril de 2020, disponível no Repositório de Arquivos do MEC (RAMEC) (BRASIL, 2020).

Para a análise da estrutura curricular foram utilizadas as informações disponíveis nos sites oficiais ou nos sistemas online de gestão acadêmica de cada IES, matrizes curriculares e Projetos Políticos Pedagógicos (PPP). Como critério de inclusão foi considerado pertencer a cursos de graduação em Educação Física ativos em IES pública brasileira, e como critério de exclusão não disponibilizar o acesso à matriz curricular ou aos programas e ementas das disciplinas em portais eletrônicos oficiais do curso ou instituição.

Para análise das matrizes curriculares foram buscadas as disciplinas que mencionassem a palavra "saúde" na nomenclatura ou outros termos que remetessem à área da Saúde Coletiva/Saúde Pública (SUS, epidemiologia, atenção primária/ básica, cuidados primários, atenção hospitalar, integralidade), bem como qualquer outro termo que indicasse vinculação da disciplina ao tema. Após a seleção inicial, foram analisados os programas e as ementas de cada disciplina, com base nas recomendações das DCN para graduação em Educação Física (BRASIL, 2018a) e nas orientações do CNS para a formação nos cursos da área da Saúde (BRASIL, 2018c), emergindo as categorias de análise: 1. Conteúdo; 2. Inserção prática no SUS e 3. Carga horária em Saúde Coletiva. 


\section{RESULTADOS E DISCUSSÃO}

Analisamos 172 currículos ativos e regulares de graduação em Educação Física de 87 IES do Brasil: 106 licenciaturas (61\%) e 66 bacharelados (39\%); 154 apresentaram disciplinas com temática ligada à área da Saúde Coletiva; 19 (10,5\%) dos cursos de Educação Física em IES públicas não apresentam nenhuma disciplina que contemple a temática, 17 licenciaturas e 2 bacharelados.

A total ausência da discussão sobre Saúde Coletiva na estrutura curricular de bacharelado em IES públicas é bastante preocupante, ainda que apenas em dois cursos, visto o reconhecimento, desde 1997, da profissão como integrante da área e a primeira DCN para graduação em Educação Física prever conteúdos da atenção à saúde como parte das competências gerais deste profissional (BRASIL, 2002).

Em currículos de licenciatura, apesar de não conflitar com o que é proposto pela nova DCN (BRASIL, 2018a), que define claramente o campo de atuação do licenciado apenas na educação básica, a ausência de discussões sobre os conteúdos da saúde nas disciplinas reflete o desconhecimento ou o descaso a respeito da discussão para a educação, desconsideram as ações de saúde como parte do currículo escolar e reforçam a formação fragmentada, parcial e reduzida, com decorrências na identidade profissional que tem sido produzida e reproduzida a partir do mercado de trabalho que se perpetua e a formação e a atuação profissional que precisaria se rever e se reinventar no ambiente universitário, não tem força e, portanto, não encontra espaço para contrapor o modus operandi.

Para a análise das matrizes curriculares, foram avaliadas as ementas e/ ou programas das disciplinas de saúde identificadas, observando os conteúdos abordados, a inserção prática no SUS e a carga horária em Saúde Coletiva distribuída ao longo da formação. Nesta etapa foram avaliados 132 cursos que disponibilizaram publicamente as ementas e programas dos componentes, estando 22 cursos (11 licenciaturas e 11 bacharelados) excluídos da análise pela indisponibilidade de acesso às ementas e programas.

\subsection{CONTEÚDOS}

Os conteúdos identificados nas matrizes curriculares foram elencados e foi criado um quadro de referência dos temas da Saúde Coletiva, tendo como base a DCN e a Resolução CNS (BRASIL, 2018a; 2018c). A partir desse quadro (Quadro 1), identificamos lacunas e ausências na área, além de levantarmos as temáticas mais valorizadas pelas formações. Importante salientar que as temáticas elencadas aglutinam distintas abordagens teórico-metodológicas de cada um dos quinze temas, englobando as perspectivas biológico-fisiológica e também sociocríticas encontradas nos diferentes currículos. 
Quadro 1 - Quadro com os conteúdos presentes nas matrizes curriculares de graduação em Educação Física das IES públicas brasileiras.

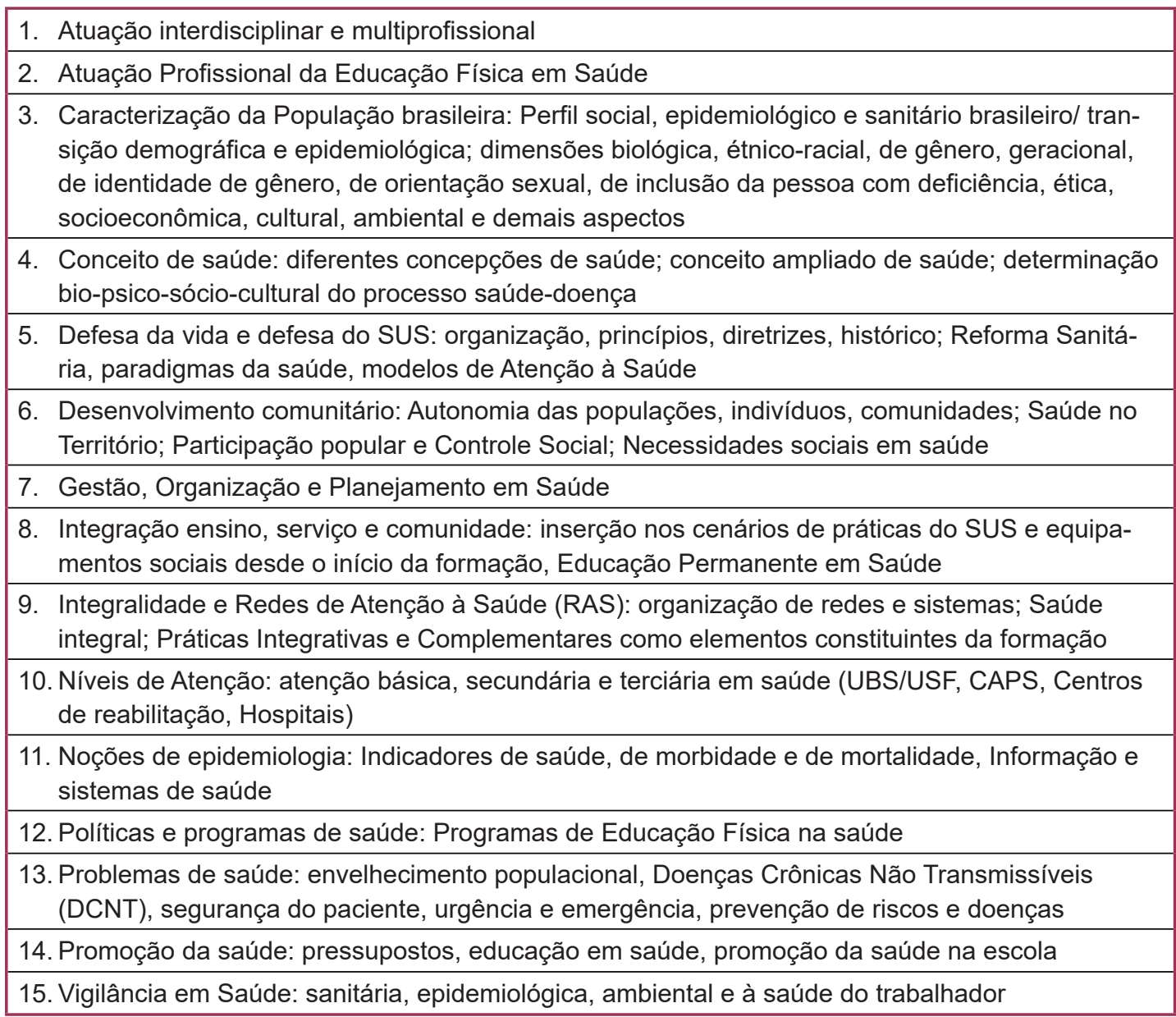

Fonte: dados da pesquisa (2021).

De acordo com a Resolução do CNS, a formação em saúde deve equilibrar conteúdos tanto em Saúde Coletiva quanto na prática clínica/assistência em saúde, além de propiciar o desenvolvimento de habilidades e atitudes coerentes com esse enfoque (BRASIL, 2018c). Por outro lado, prevê uma formação que integre serviçoensino-comunidade aproximando o estudante da realidade do SUS desde o início da formação. Dessa forma entende-se que uma formação integrando todos os conteúdos do quadro de referência estaria em acordo com essas recomendações.

No entanto, o que observamos foi uma grande heterogeneidade na distribuição dos conteúdos, havendo cursos com ofertas apenas optativas ou que atendem a temáticas transversais (direitos humanos, aspectos éticos, primeiros socorros, pessoas com deficiência ou mobilidade reduzida, educação ambiental, relações étnico-raciais), outros que atendem até sete dos 15 conteúdos $(50 \%)$ constantes no quadro de referência, e uma minoria de cursos que atendem a mais de sete conteúdos. Grande diferença ainda é observada quando comparamos licenciaturas e bacharelados, conforme pode ser observado na Tabela 1. 
Tabela 1 - Cursos de Educação Física do Brasil de IES públicas por nível de presença de conteúdos obrigatórios da Saúde Coletiva na matriz curricular, de acordo com o quadro de referência.

\begin{tabular}{cccccc}
\hline & $\begin{array}{c}\text { Nenhum } \\
\text { conteúdo } \\
\mathbf{n}(\%)\end{array}$ & $\begin{array}{c}\text { Contempla até } \mathbf{5 0 \%} \\
\text { dos conteúdos } \\
\mathbf{n}(\%)\end{array}$ & $\begin{array}{c}\text { Contempla mais } \\
\text { de } \mathbf{5 0 \% \text { dos }} \\
\text { conteúdos } \\
\mathbf{n}(\%)\end{array}$ & $\begin{array}{c}\text { Contempla } \\
\text { todos os } \\
\text { conteúdos } \\
\mathbf{n}(\%)\end{array}$ & $\begin{array}{c}\text { TOTAL } \\
\mathbf{n}(\%)\end{array}$ \\
\hline Bacharelado & $5(3,8)$ & $36(27,3)$ & $12(9,1)$ & $1(0,7)$ & $54(40,9)$ \\
Licenciatura & $15(11,4)$ & $62(47)$ & $1(0,7)$ & $0(0)$ & $78(59,1)$ \\
\hline TOTAL & $\mathbf{2 0 ( 1 5 , 2 )}$ & $\mathbf{9 8 ( 7 4 , 3 )}$ & $\mathbf{1 3 ( 9 , 8 )}$ & $\mathbf{1 ( 0 , 7 )}$ & $\mathbf{1 3 2 ( 1 0 0 )}$ \\
\hline
\end{tabular}

Fonte: dados da pesquisa (2021).

Observamos uma diferença importante entre bacharelados e licenciaturas: os currículos das licenciaturas apresentam majoritariamente menos de $50 \%$ dos conteúdos, enquanto os currículos com mais de $50 \%$ dos conteúdos são quase unanimemente bacharelados. Esse dado confirma uma diferenciação entre as duas formações, já enfatizada por estudos anteriores, em que a licenciatura apresenta um distanciamento da Saúde Coletiva marcada pela menor oferta de disciplinas quando comparada aos bacharelados (ANJOS; DUARTE, 2009; BRUGNEROTTO; SIMÕES, 2009; COSTA et al., 2012; OLIVEIRA; GOMES, 2020).

Esse achado evidencia um entendimento da formação em Educação Física bastante atrelado ao campo de atuação profissional fragmentado, em que os currículos de licenciatura deveriam privilegiar a área da educação, ao passo que o bacharelado priorizaria a saúde, o esporte e o lazer. Loch, Rech e Costa (2020) salientam, entretanto, a necessidade de superar essa ideia comum da saúde como temática exclusiva da formação dos bacharéis, e da mesma forma Oliveira e Gomes (2020) trazem que a nova DCN reforça a fragmentação profissional ao reafirmar explicitamente atuação na saúde como campo do bacharel e não fazer nenhuma menção à temática na formação do licenciado, fato este que pode obscurecer as discussões sobre saúde nos currículos de licenciaturas.

No entanto, é necessário ressaltar que ambas as formações apresentam, de modo geral, insuficientes conteúdos da Saúde Coletiva, sendo o bacharelado da Universidade Federal de Goiás, campus de Goiânia, o único a agregar todos os conteúdos elencados. Cabe mencionar ainda que a distribuição desses conteúdos nos currículos também não se deu de maneira homogênea, de modo que alguns são priorizados em detrimento de outros, conforme observa-se na Figura 1. 
Figura 1 - Conteúdos de Saúde Coletiva presentes nas matrizes curriculares de licenciaturas e bacharelados de IES públicas brasileiras

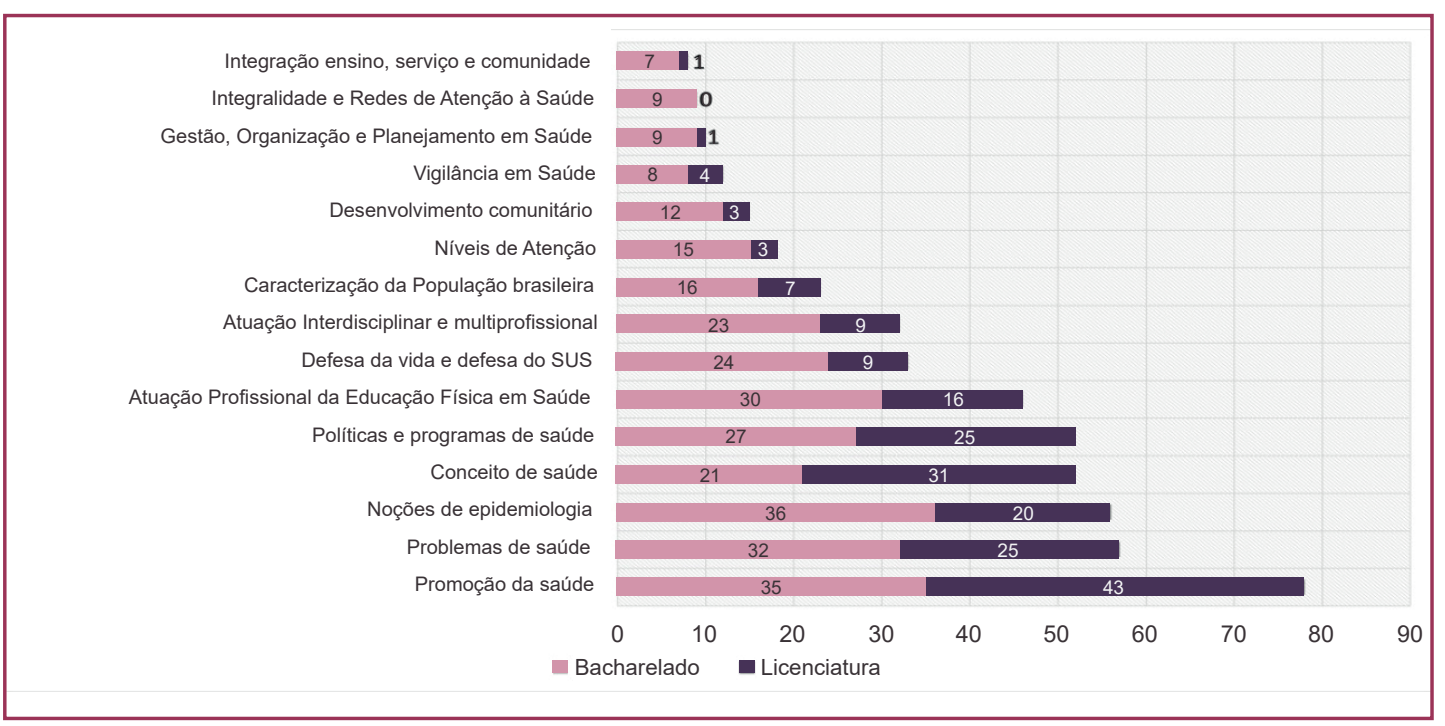

Fonte: dados da pesquisa (2021).

Existe diferença entre predominância de temas entre as duas modalidades. Nas licenciaturas, predominam os temas ligados à promoção da saúde (com foco na promoção da saúde escolar), ao conceito de saúde, aos problemas de saúde (com foco na saúde de escolares) e às políticas públicas, principalmente relacionando saúde e educação.

Há baixa prevalência de conteúdos relacionados ao SUS e à atuação profissional nesse espaço (defesa da vida e defesa do SUS; atuação interdisciplinar e multiprofissional; caracterização da população brasileira; vigilância em saúde; níveis de atenção; desenvolvimento comunitário; gestão, organização e planejamento em saúde; integração ensino, serviço e comunidade; integralidade e redes de atenção à saúde - temas contemplados em menos de $15 \%$ dos cursos avaliados).

Os bacharelados, por sua vez, priorizam conteúdos ligados às noções de epidemiologia, promoção da saúde (com foco na prevenção de doenças e promoção da qualidade de vida) e problemas de saúde (priorizando a relação entre exercício físico e doenças crônicas). Entre os conteúdos menos priorizados estão os de gestão, organização e planejamento em saúde; integralidade e redes de atenção à saúde; vigilância em saúde; e integração ensino, serviço e comunidade evidenciando maior valorização de abordagens teórico-conceituais, com baixa integração com a prática e de uma perspectiva de cuidado individual; centrada no profissional; e nos exercícios físicos à medida que pouco valorizam o pensamento voltado para o cuidado em rede, a vigilância em saúde, a gestão e o planejamento dos serviços e da atenção à saúde.

A predominância de temas ligados à promoção da saúde, noções de epidemiologia e problemas de saúde revela uma tendência observada pela literatura, em que existe uma noção de saúde na Educação Física ainda fortemente ligada a elementos biológicos, qualidade de vida, aptidão física e bem-estar (ANJOS; DUARTE, 2009; BRUGNEROTTO; SIMÕES, 2009; OLIVEIRA; GOMES, 2019; OLIVEIRA; GOMES, 2020). 
Loch, Rech e Costa (2020) apontam que a área tem se concentrado em discussões sobre aptidão física e exercício físico como fator de promoção da qualidade de vida e tem negligenciado conhecimentos fundamentais em saúde como medidas sanitárias e epidemiologia, situação atribuída aos posicionamentos conflitantes emitidos por conselhos de classe e profissionais de Educação Física, especialmente visível durante a pandemia de covid-19, em que diante do número crescente de mortes pelo vírus, foi pedida a reabertura das academias sob a justificativa dos benefícios da prática regular de exercícios para a saúde.

Esse tipo de associação causal e rasa entre atividade física e saúde, ainda bastante prevalente nas formações, e a ausência de inserção prática no SUS, com valorização dos espaços privados como cenários privilegiados de prática para os estudantes apenas aprofundam essa percepção e fragilizam a formação para atuação no campo da Saúde Coletiva.

A necessidade de aproximação com o SUS vem sendo pautada em pesquisas que se dedicam a compreender aspectos da formação. Fraga, Gomes e Carvalho (2012) sinalizaram a ausência de menção ao SUS nas DCN vigentes na Educação Física. Apesar da versão mais atual da DCN incluir o SUS no eixo da saúde para a formação dos bacharéis, a materialização nos currículos ainda acontece de maneira tímida. Levando em consideração que a DCN foi publicada em dezembro de 2018 e atribuiu um prazo de dois anos para a adequação dos currículos ativos e que 2020 foi um ano atípico devido à pandemia de covid-19, as reformulações curriculares a fim de atender a essas novas proposições devem ocorrer em um futuro próximo.

\subsection{INSERÇÃO PRÁTICA NO SUS}

Os documentos orientadores recomendam a inserção prática no SUS como parte da formação em saúde com diversificação de cenários de práticas, possibilitando aos discentes vivenciar as políticas de saúde, os fluxos de atenção em rede e de organização do trabalho em equipe interprofissional (BRASIL, 2018c).

O que apareceu de forma predominante, tanto nos cursos de licenciatura quanto de bacharelado investigados, foi a ausência total de inserção no SUS, porém se destacando nos bacharelados um contingente maior de cursos que garantem o serviço público de saúde como campo de prática, conforme observa-se na Tabela 2.

Tabela 2 - Inserção prática no SUS nos cursos de graduação em Educação Física de IES públicas

\begin{tabular}{ccccc}
\hline & $\begin{array}{c}\text { Ausência de } \\
\text { inserção no SUS } \\
\mathbf{n}(\%)\end{array}$ & $\begin{array}{c}\text { Não explicita carga } \\
\text { horária prática no SUS } \\
\mathbf{n}(\%)\end{array}$ & $\begin{array}{c}\text { Explicita carga horária } \\
\text { prática no SUS } \\
\mathbf{n}(\%)\end{array}$ & $\begin{array}{c}\text { TOTAL } \\
\mathbf{n}(\%)\end{array}$ \\
\hline Licenciatura & $71(53,8)$ & $2(1,5)$ & $5(3,8)$ & $78(59,1)$ \\
Bacharelado & $30(22,7)$ & $9(6,8)$ & $15(11,4)$ & $54(40,9)$ \\
\hline TOTAL & $\mathbf{1 0 1 ( 7 6 , 5 )}$ & $\mathbf{1 1 ( 8 , 3 )}$ & $\mathbf{2 0 ( 1 5 , 2 )}$ & $\mathbf{1 3 2 ( 1 0 0 )}$ \\
\hline
\end{tabular}

Fonte: dados da pesquisa (2021).

A necessidade da explicitação dos cenários de práticas e dos compromissos com a interprofissionalidade nos componentes curriculares e PPP é sinalizada pelo 
documento do CNS (BRASIL, 2018c), como forma de garantia da valorização da inserção e formação para o SUS. Foi observado, no entanto, que 11 cursos trouxeram nos programas de disciplinas a inserção prática na saúde sem nomear os serviços do SUS como campo de inserção. Esse tipo de lacuna permite que academias, clubes e outros espaços privados possam ser priorizados como campo de atuação em saúde e o estudante se forme sem experienciar nada do serviço público.

A inserção no início da formação é outra recomendação do CNS (BRASIL, 2018c) como estratégia de integrar a educação-trabalho e saúde, porém, analisando as ementas e programas, apenas em quatro graduações identificamos esse contato com os serviços do SUS no primeiro ano da formação. Em dois cursos de licenciatura, da Universidade do Estado da Bahia - campi Guanambi e Jacobina -, e dois bacharelados, na Universidade Estadual de Maringá e Universidade Federal de São Paulo.

Essa inserção antecipada é uma forma de garantir que os estudantes se aproximem do campo do SUS logo no início do curso com intuito de conhecerem o SUS por dentro e se apropriarem da complexidade do Sistema por sucessivas aproximações ao longo da formação. Cabe mencionar, no entanto, que a inserção inicial sem uma vivência longitudinal de SUS e conteúdos da Saúde Coletiva não têm grande efeito no processo formativo. É o que ocorre nas duas licenciaturas mencionadas: ao longo do curso não são identificadas outras disciplinas que dialoguem com o campo da Saúde Coletiva, portanto não oferecem as bases necessárias, transformam a inserção prática em experiência pontual e isolada.

\subsection{CARGA HORÁRIA EM SAÚDE COLETIVA}

A Resolução CNS (BRASIL, 2018c) não explicita a carga horária para os conteúdos de saúde, recomendando apenas o total de 4.000 horas para os cursos de saúde, enquanto a DCN da Educação Física recomenda um mínimo de 3.200 horas (BRASIL, 2018a).

Neste estudo as cargas horárias totais dos cursos de bacharelado tiveram uma média de 3.374 horas, enquanto as licenciaturas apresentaram média de 3.268 horas, porém com diferenças no espaço reservado à Saúde Coletiva nos currículos das licenciaturas e bacharelados, conforme verifica-se na Tabela 3.

Tabela 3 - Carga horária em Saúde Coletiva nos cursos de graduação em Educação Física de IES públicas brasileiras.

\begin{tabular}{cccccc}
\hline & Optativas & $<90 h$ & $\geq 90 h ;<200 h$ & $\geq 200 h$ & TOTAL \\
\hline Licenciatura & 7 & 54 & 17 & 0 & 78 \\
Bacharelado & 2 & 17 & 14 & 21 & 54 \\
\hline TOTAL & $\mathbf{9}$ & $\mathbf{7 1}$ & $\mathbf{3 1}$ & $\mathbf{2 1}$ & $\mathbf{1 3 2}$ \\
\hline
\end{tabular}

Fonte: dados da pesquisa (2021).

As matrizes curriculares que tiveram carga horária obrigatória em Saúde Coletiva abaixo de 90 horas apresentaram, de maneira geral, a condensação em 
uma única disciplina de caráter teórico com ausência de inserção na prática no SUS e dispostas nos anos finais da formação. Grande parte das matrizes de licenciaturas se concentraram nesse perfil, evidenciando uma formação bastante pontual e que em nada atende às $D C N$.

Os cursos agrupados entre 90 e 200 horas apresentam carga horária distribuída de duas a três disciplinas, sem integração entre os conteúdos e disposta nos anos finais da formação. Uma menor quantidade de cursos (quatro bacharelados e cinco licenciaturas) apresenta carga horária prática no SUS, porém os demais restringem a uma abordagem apenas teórica da saúde. São essas licenciaturas a da Universidade do Estado da Bahia - campi Teixeira de Freitas, Guanambi e Jacobina -, Universidade Federal da Bahia e Instituto Federal de Educação, Ciência e Tecnologia do Ceará campus Canindé.

A disciplina ofertada pela IES cearense é de prática curricular, obrigatória do $6^{\circ}$ período, tendo por objetivo aproximação com campos de atuação da promoção da saúde, incluindo além do ambiente escolar, campos do SUS. Já as IES baianas apresentam disciplinas de Estágio Supervisionado obrigatórias no $7^{\circ}$ semestre do curso, com carga horária entre 75-100 horas contemplando atuação supervisionada do estudante de licenciatura nos serviços de saúde. Essa tendência identifica a luta histórica dessas instituições contra a fragmentação profissional em licenciatura e bacharelado e em favor da reunificação da formação, tendo como um outro reflexo a inexistência de cursos de bacharelado ativos nas IES públicas do estado até o presente, uma vez que a defesa política que se faz é de uma licenciatura plena de caráter ampliado (TAFFAREL, 2012).

Os cursos identificados com carga horária obrigatória em Saúde Coletiva acima de 200 horas reuniu exclusivamente bacharelados, que apresentaram uma distribuição temática ao longo dos quatro anos de formação e com disciplinas práticas e teóricas. Quatro cursos apresentaram carga horária em saúde superior a 400h (Universidade Federal de Goiás - campus Goiânia -, Universidade Federal de São Paulo, Universidade Federal de Pernambuco, Universidade do Estado de Minas Gerais - campus Divinópolis). Apesar de a garantia de carga horária não ser marca de qualidade na formação e nem de preparo para atuação no SUS, a reserva de espaço no currículo para a temática indica avanço.

Outra tendência importante observada foram currículos de algumas IES com ênfase em áreas temáticas (saúde, esporte, lazer). Esse tipo de formação é recomendado pela Associação Brasileira de Ensino da Educação Física para a Saúde (ABENEFS), que espera a atenuação de desequilíbrios regionais e acadêmicos pelo impacto da formação de egressos com maior arcabouço em saúde (FONSECA et al., 2012).

Os currículos identificados com ênfase em saúde (bacharelados da Universidade Federal de São Paulo, Universidade de São Paulo - campus Zona Leste -, Universidade Federal do Rio Grande do Sul, Universidade Federal de Santa Catarina, Universidade Estadual de Londrina, Universidade Federal de Juiz de Fora) apresentaram carga horária em saúde acima de 200h e distribuída ao longo de todo o curso. Porém, a multiplicidade e as divergências sobre o conceito de saúde presente 
nos currículos são uma questão a ser observada, uma vez que apenas um deles apresentou a inserção e a prática em saúde direcionada explicitamente pelo e para o SUS (UNIFESP). Importante observar que a ampliação e o direcionamento para a área da saúde não são suficientes, pois uma vez que se estruturam cursos em uma concepção curativista ou voltada aos interesses privados, a grande lacuna formativa para atuar no SUS permanecerá.

Ainda é importante ressaltar que as formações com ênfase abrem brechas para que se formem profissionais de Educação Física dos demais núcleos temáticos com baixa vivência em saúde e que podem, uma vez que não há restrições legais e normativas, vir a ocupar cargos e funções no SUS, reforçando as fragilidades da atuação profissional. Esta acaba não sendo, portanto, uma solução, mas apenas mais uma forma de fragmentar a formação.

Alguns autores têm apontado para a necessidade de instituir a Saúde Coletiva como temática transversal nos currículos de Educação Física, como alternativa para essas ausências e insuficiências, visando fortalecer a formação para o SUS (ABENEFS, 2016; PASQUIM, 2010). No entanto, a construção de currículos é um processo coletivo marcado por disputas de saberes, identidades e poder, que reflete os diferentes projetos de sociedade dos atores envolvidos na sua construção, e que muitas vezes se constitui como espaço de legitimação das práticas dos docentes. Nessa perspectiva, o produto final dessa disputa tende a preservar as concepções pautadas em determinada tradição da Educação Física estabelecidas em um espaço já legitimado, tornando um desafio a construção de um projeto comum orientado ética, estética e politicamente para uma perspectiva contra-hegemônica de saúde, como a Saúde Coletiva (ALVIANO JR; NEIRA, 2017).

Para além disso, a transversalização do tema não garante a superação de uma visão reducionista de saúde e corpo. Madel Luz (2007) destaca que a formação em Saúde Coletiva dos profissionais de Educação Física não deve se restringir a uma "consciência sanitária", e isso passa por pensar a saúde, vida e vitalidade não apenas pela dimensão física, biomecânica, mas requer um reposicionamento da função de treinar, adestrar, habilitar, tornar apto, para um novo arranjo em que dimensões cognitivas, sensoriais, emocionais, sociais e espirituais estejam contempladas. A experimentação, a busca por outras percepções e concepções de corpo, movimento, saúde, vitalidade e autonomia são apontadas como necessárias para o ensino na graduação que prepare o estudante para esse encontro com as complexidades e diversidades humanas existentes.

\subsection{PERSPECTIVAS E AVANÇOS}

A insuficiente formação na área específica no que se refere à Saúde Coletiva não é recente e as necessidades encontradas neste trabalho (prevalência de formação curativista e biológica, sem inserção no SUS e com carga horária inadequada) têm sido apontadas extensamente por outros autores. Mas, apesar do cenário bastante distante do que se almeja para o trabalho na saúde pública, é necessário reconhecer que muito já se conquistou e que essas lacunas são provenientes de disputas de modelos e as mudanças exigem tempo. 
Anjos e Duarte (2009) apontam que o curso de Educação Física na Universidade Federal de São Carlos, até o momento da publicação, ofertava apenas a modalidade licenciatura e não oferecia disciplinas abordando Saúde Pública, nem Saúde Coletiva e tampouco estágios em serviços de saúde. Os achados neste trabalho evidenciam uma diferença na instituição, que hoje conta com as duas modalidades e no bacharelado a carga horária obrigatória em saúde é acima de $200 \mathrm{~h}$, abordando temáticas da saúde pública e Saúde Coletiva e garantindo vivências e inserção no SUS.

Ainda que existam diferenças entre as análises documentais e as que ocorrem na micropolítica das IES e mesmo considerando a ausência dessas análises in loco com os atores sociais da UFSCar que nos ajudassem a pensar as verdadeiras modificações que ocorreram nos últimos dez anos, não se podem negar os avanços em termos de estrutura curricular e, portanto, dos impactos que isso vem desencadeando nos profissionais formados pela instituição nesse período.

Para além disso, existem experiências formativas nas universidades brasileiras que têm se destacado e sido evidenciadas pela defesa que fazem desse alinhamento da formação em Educação Física com os princípios e diretrizes do SUS e pelo esforço em superar muitos desses limites apontados anteriormente.

O bacharelado da Universidade Federal de São Paulo, campus Baixada Santista, é uma dessas experiências. Presente em uma instituição que tem por pressuposto teórico e epistemológico a educação interprofissional na graduação em saúde, a UNIFESP abriga, além da Educação Física, outros seis cursos de graduação, todos eles estruturados sobre três eixos comuns a todos os cursos: ser humano e sua dimensão biológica; o ser humano e sua inserção social; e trabalho em saúde, e um eixo específico por área relacionada à prática específica em saúde. Assim, os estudantes das áreas de saúde têm ao longo do curso uma vivência formativa com outras áreas da saúde, inclusive realizando inserção no SUS no início da formação com essa perspectiva de oportunizar contextos científicos e acadêmico-institucionais para o encontro com a interdisciplinaridade através do ensino interprofissional (UNIVERSIDADE FEDERAL DE SÃO PAULO, 2019).

O formato disciplinar nos currículos encontra raízes no processo historicamente construído de especialização do conhecimento científico, e a interdisciplinaridade é apontada como solução a essa fragmentação, na perspectiva de dar conta da complexificação dos problemas na modernidade através da superação das barreiras entre os diversos conteúdos (AIRES, 2011). Isso já nos ajuda a pensar que existem limites que a simples inclusão de disciplinas nos currículos não é capaz de transpor, e que para atender às "complexas e dinâmicas necessidades de saúde" (BRASIL, 2018c) a aposta que tem sido feita é no sentido de fortalecer a interdisciplinaridade entre os conteúdos.

A construção dessa interdisciplinaridade, no entanto, é bastante desafiadora, aparecendo muitas vezes nos currículos como conteúdo de algumas disciplinas, porém, enquanto prática, de maneira muito escassa. Nos currículos avaliados, a grande maioria dos cursos $(75,7 \%, n=100)$ não inclui a interdisciplinaridade como conteúdo; dos que incluem, grande parte $(93,7 \%, n=31)$ limita-se à discussão apenas 
teórica a respeito da atuação profissional, porém as dimensões do trabalho e práticas interdisciplinares e a própria configuração curricular não avança.

A experiência da UNIFESP, que busca a ruptura com os modelos disciplinares rígidos pela integração de diferentes conhecimentos, áreas disciplinares e profissionais, se destaca na construção de uma proposta curricular inovadora na Educação Física, e por estar em acordo com as recomendações da nova DCN e Resolução CNS (BRASIL, 2018a; 2018c), pode inspirar as reformulações curriculares que devem se seguir nos próximos anos.

\section{CONSIDERAÇÕES FINAIS}

Permanece na Educação Física grande defasagem na formação no que se refere ao diálogo e à composição com a Saúde Coletiva. Este estudo evidencia que, quando presente de maneira obrigatória nos currículos, as disciplinas da área de saúde se apresentam como componentes isolados, que não se articulam com outras disciplinas. Os conteúdos que predominam estão ligados às áreas biológicas, têm carga horária limitada e não há inserção e diálogo com o SUS. As lacunas mais severas foram identificadas nas formações em licenciatura.

Os avanços têm se dado ainda de maneira insuficiente, e mesmo com as intensas modificações que a área sofreu desde o seu reconhecimento como profissão da saúde pelo CNS, com a inserção do profissional de Educação Física em diferentes políticas públicas, ainda se faz necessário fortalecer a Saúde Coletiva e o SUS enquanto eixos orientadores da formação.

O rompimento com a hegemonia das perspectivas uniprofissional, privatista e biológica; a aposta no SUS como campo de formação e integração com os serviços de saúde, com usuários e gestores do sistema; e a abertura de espaço para as ciências políticas, humanas e sociais nos currículos são realidades ainda distantes e continuarão a se perpetuar nos currículos enquanto não houver resposta efetiva e comprometida com a DCN de 2018 no que se refere ao SUS e à Saúde Coletiva na formação em Educação Física.

Experiências têm ocorrido no sentido de tentar mitigar essas lacunas: a criação de currículos com ênfase; formações interdisciplinares e interprofissionais em saúde; iniciativas via extensão universitária; o programa PET-Saúde e Pró-PET-Saúde; e as residências multiprofissionais podem ser exemplos e vêm se mostrando como avanços importantes no enfrentamento das resistências e dos impasses e precisam ser fortalecidas.

Outras experiências de formação, que extrapolam as dimensões curriculares investigadas neste trabalho, também merecem investigações futuras, como o movimento estudantil, a extensão universitária, os grupos e mobilizações comunitárias e outros projetos de docentes, discentes e da própria comunidade. Importante também futuras investigações que incluam as IES particulares, não contempladas neste estudo, mas que hoje se configuram como crescente porta de entrada ao Ensino Superior para muitos estudantes. 
Necessário destacar ainda como limites deste trabalho a escolha das disciplinas a serem analisadas a partir dos títulos, e não dos seus programas e ementas, podendo haver disciplinas que não foram incluídas nas análises. A própria análise da formação a partir das disciplinas já se configura um limite, sendo necessários outros estudos a partir dos PPP dos cursos que venham a complementar os resultados aqui encontrados.

Espera-se que nos próximos anos seja possível acompanhar intensas reformulações curriculares em todos os cursos de Educação Física visando atender a nova DCN de 2018, bem como a nova realidade de saúde decorrente da pandemia de covid-19. Entendemos que nosso trabalho contribui no sentido de fortalecer a Saúde Coletiva nos currículos, integrando ensino e serviço, aproximando os estudantes dos diferentes cenários do SUS, da atenção básica, orientados pela visão ampliada de saúde e pelo cuidado interdisciplinar, interprofissional e comunitário.

Deixamos aqui um ousado e desafiador convite para a área: promovermos encontros para pensar a formação que queremos e a que praticamos a fim de definir novos e diferentes rumos para a Educação Física, sobretudo trabalhando com os projetos políticos pedagógicos de modo compartilhado nesse momento tão propício também para as discussões relativas à formação e aos currículos na área específica.

\section{REFERÊNCIAS}

ABENEFS. Manifesto da ABENEFS: alinhando a formação inicial em Educação Física às necessidades do setor saúde. Revista Brasileira de Atividade Física e Saúde, v. 21, n. 2, p.105-109, 2016.

AIRES, Joanes. Integração curricular e interdisciplinaridade: sinônimos? Educação e Realidade, v. 36, n.1, p. 215-230, jan./abr. 2011.

ALVIANO JR, Wilson; NEIRA, Marcos Garcia. Formação inicial em Educação Física: análises de uma construção curricular. Educação em Foco, v. 22, n. 1, p. 1-32, jun. 2017.

ANJOS, Tatiana Coletto dos; DUARTE, Ana Cláudia Garcia de Oliveira. A Educação Física e a Estratégia de Saúde da Família: formação e atuação profissional. Physis - Revista de Saúde Coletiva, v. 19, n. 4, p. 1127-1144, 2009.

BRASIL. Conselho Nacional de Saúde. Resolução n. 218, de 6 de março de 1997. Diário Oficial da União, Brasília, 5 de maio 1997, Seção 1, n. 83, p. 8932-33, 1997.

BRASIL. Conselho Nacional de Saúde. Resolução n 569, de 8 de dezembro de 2017.

Diário Oficial da União, Brasília, 26 de fevereiro de 2018, Seção 1, p. 85-90, 2018c.

BRASIL. Ministério da Educação. Base nacional comum curricular. Brasília: MEC, 2018b. Disponível em: http://basenacionalcomum.mec.gov.br/. Acesso em: 10 out. 2021.

BRASIL. Ministério da Educação. Lista de cursos superiores de graduação e sequenciais cadastro e-MEC. Brasília: MEC, 15 de abril, 2020. Disponível em: http:// ramec.mec.gov.br/seres/6950-lista-de-cursos-superiores-de-graduacao-e-sequenciaiscadastro-e-mec-expedicao-em-15-04-2020. Acesso em: 31 jul. 2020. 
BRASIL. Ministério da Educação. Conselho Nacional de Educação. Parecer CNE/CES 138/2002, de 3 de abril de 2002. Institui diretrizes curriculares do curso de graduação em Educação Física. Brasília: MEC, 2002. Disponível em: http://portal.mec.gov.br/sesu/ arquivos/pdf/13802EdFisica.pdf. Acesso em: 11 fev. 2021.

BRASIL. Ministério da Educação. Conselho Nacional de Educação. Câmara de Educação Superior. Institui diretrizes curriculares nacionais dos cursos de graduação em Educação Física e dá outras providências. Resolução n. 6, de 18 de dezembro de 2018. Diário Oficial da União, Brasília, 19 de dezembro de 2018, Seção 1, p. 48-49, $2018 a$.

BRASIL. Ministério da Educação. Secretaria de Educação Fundamental. Parâmetros curriculares nacionais: Educação Física/ Secretaria de Educação Fundamental. Brasília: MEC,1998.

BRUGNEROTTO, Fabio; SIMÕES, Regina. Caracterização dos currículos de formação profissional em Educação Física: um enfoque sobre saúde. Physis - Revista de Saúde Coletiva, v. 19, n. 1, p. 149-172, 2009.

COSTA, Larissa Chaves; LOPES JUNIOR, Carlos Alberto Furtado; COSTA, Edmara Costa; FEITOSA, Michelle Cochrane; AGUIAR, Jaina Bezerra de; GURGEL, Luilma Albuquerque. Formação profissional e produtividade em saúde coletiva do profissional de Educação Física. Revista Brasileira de Atividade Física e Saúde, v. 17, n. 2, p.107-113, abr. 2012.

FONSECA, Silvio Aparecido; MENEZES, Aldemir Smith; FEITOSA, Wallacy Milton do Nascimento; LOCH, Mathias Roberto. Notas preliminares sobre a Associação Brasileira de Ensino da Educação Física para a saúde - Abenefs. Caderno Fnepas, v. 2, p. 38-48, 2012.

FRAGA, Alex Braga; GOMES, Ivan Marcelo; CARVALHO, Yara Maria de. Políticas de formação em Educação Física e Saúde Coletiva. Trabalho, Educação e Saúde, v. 10, n. 3 , p. 367-386, 2012.

HADDAD, Ana Estela; MORITALL, Maria Celeste; PIERANTONI, Célia Regina; BRENELLI, Sigisfredo Luis; PASSARELLA, Teresa; CAMPOS, Francisco Eduardo. Formação de profissionais de saúde no Brasil: uma análise no período de 1991 a 2008. Revista de Saúde Pública, v.44, n. 3, p. 383-393, 2010. Disponível em: https://www.scielosp.org/pdf/ rsp/2010.v44n3/383-393/pt. Acesso em: 10 set. 2020.

LOCH, Mathias Roberto; RECH, Cassiano Ricardo; COSTA. Filipe Ferreira da. A urgência da Saúde Coletiva na formação em Educação Física: lições com o COVID-19. Ciência e Saúde Coletiva, v. 25, n. 9, p. 3511-3516, jun. 2020. Disponível em: https://www.scielo.br/j/ csc/a/GFfQWspRqzRHPzYMm7k6V7K/?lang=pt\&format=pdf. Acesso em: 13 jun. 2020.

LUZ, Madel Terezinha. Educação física e saúde coletiva: papel estratégico da área e possibilidades quanto ao ensino na graduação e integração na rede de serviços públicos de saúde. In: FRAGA, Alex Branco; WACHS, Felipe. (orgs.). Educação Física e saúde coletiva: políticas de formação e perspectivas de intervenção. Porto Alegre: UFRGS, 2007. p. 33-46.

MANOEL, Edison de Jesus; CARVALHO, Yara Maria de. Pós-graduação na Educação Física brasileira: a atração (fatal) para a biodinâmica. Educação e Pesquisa, v. 37, n. 2, p. 389-406, maio/ago. 2011.

OLIVEIRA, Rogério Cruz; ANDRADE, Douglas Roque. Formação profissional em Educação Física para o setor da saúde e as diretrizes curriculares nacionais. Pensar a Prática, v. 19, n. 4, out./dez. 2016. 
OLIVEIRA, Victor José Machado; GOMES, Ivan Marcelo. A saúde nos currículos de Educação Física em uma universidade pública. Trabalho, Educação e Saúde, v. 18, n. 3, 2020.

OLIVEIRA, Victor José Machado; GOMES, Ivan Marcelo. Caracterização episódica sobre o tema da saúde nos currículos de formação em Educação Física em uma universidade federal pública. Motrivivência, v. 31, n. 60, p. 01-24, out./dez. 2019.

PASQUIM, Heitor Martins. A saúde coletiva nos cursos de graduação em Educação Física. Saúde e Sociedade, v.19, n.1, p.193-200, 2010.

TAFFAREL, Celi Nelza Zulke. A Formação de professores de Educação Física e a licenciatura ampliada. In: SEMANA DE EDUCAÇÃO FÍSICA/UFMS, 17, 2012, Campo Grande (MS); JORNADA DE INICIAÇÃO CIENTÍFICA DO CURSO DE EDUCAÇÃO FÍSICA/ UFMS, 4., 2012, Campo Grande (MS). 38 f. [Anais]. Campo Grande: [s.n.], 2012. Disponível em: https://repositorio.ufba.br/ri/handle/ri/18055. Acesso em: 13 out. 2020.

UNIVERSIDADE FEDERAL DE SÃO PAULO. Projeto pedagógico do curso de bacharelado em Educação Física. 2021. Disponível em: https://www.unifesp.br/reitorial prograd/pro-reitoria-de-graduacao/cursos/informacoes-sobre-os-cursos. Acesso em: 10 out. 2021. 
Abstract: This study presents a national analysis of higher education in Public Health in the undergraduate schools of Physical Education of public universities. We carried out a document analysis of curricular structures, syllabuses and programs of courses offered by these universities, evaluating the contents, practical insertion in SUS, and the workload in Public Health. We analyzed 173 active and regular undergraduate curricula from 87 public institutions and gaps were found in health education: when mandatory, Public Health courses are presented as isolated components; content related to biological sciences predominates; they have limited hours and lack insertion in and dialogue with health services of SUS. Some curricular proposals have been identified that advance in health education, but still in a very timid way, especially in view of the urgency that the new reality resulting from the pandemic of COVID-19 demands from health courses.

Keywords: Physical Education. Education, higher. Public Health. Unified Health System.

Resumen: Este estudio presenta un análisis nacional de la educación superior en Salud Colectiva en los cursos de grado en Educación Física de instituciones de enseñanza superior públicas. Se realizó un análisis documental de las estructuras curriculares, contenidos y programas de disciplinas ofrecidos por esas universidades, evaluando los contenidos, la inserción práctica en el Sistema Único de Salud (SUS) y la carga horaria en Salud Colectiva. En total, se analizaron 173 currículos de grado activos y regulares de 87 instituciones públicas, y se observaron lagunas en la formación en salud: cuando son obligatorias, las disciplinas de Salud Colectiva se presentan como componentes aislados; predominan los contenidos relacionados con las ciencias biológicas; tienen una carga horaria limitada y carecen de inserción y diálogo con los servicios de salud del SUS. Se han identificado algunas propuestas curriculares que avanzan en la formación en salud, pero aún de manera muy tímida, especialmente ante la urgencia que la nueva realidad derivada de la pandemia de COVID-19 demanda a los cursos de salud.

Palabras clave: Educación Física. Educación superior. Salud Pública. Sistema Único de Salud. 


\section{LICENÇA DE USO}

Este é um artigo publicado em acesso aberto (Open Access) sob a licença Creative Commons Atribuição 4.0 Internacional (CC BY 4.0), que permite uso, distribuição e reprodução em qualquer meio, desde que o trabalho original seja corretamente citado. Mais informações em: https://creativecommons.org/licenses/by/4.0

\section{CONFLITO DE INTERESSES}

Os autores declararam que não existe nenhum conflito de interesses neste trabalho.

\section{CONTRIBUIÇÕES AUTORAIS}

Viviana Graziela de Almeida Vasconcelos Barboni: Conceituação, Curadoria de dados, Análise Formal, Aquisição de financiamento, Investigação, Metodologia, Escrita - rascunho original, Escrita - revisão e edição.

Yara Maria de Carvalho: Análise Formal, Aquisição de financiamento, Metodologia, Administração de projetos, Supervisão, Visualização, Escrita - revisão e edição.

Vagner Herculano de Souza: Análise Formal, Metodologia, Administração de projetos, Supervisão, Escrita - rascunho original, Escrita - rascunho original, Escrita - revisão e edição.

\section{FINANCIAMENTO}

O presente trabalho foi realizado com apoio da Coordenação de Aperfeiçoamento de Pessoal de Nível Superior - Brasil (CAPES) - Código de Financiamento 001. This study was financed in part by the Coordenação de Aperfeiçoamento de Pessoal de Nível Superior - Brasil (CAPES) - Finance Code 001

\section{COMO REFERENCIAR}

BARBONI, Viviana Graziela de Almeida Vasconcelos; CARVALHO, Yara Maria de SOUZA, Vagner Herculano de. A formação em saúde coletiva nos currículos de educação física: um retrato atual. Movimento (Porto Alegre), v.27, p.e27065, jan./dez. 2021. Disponível em: https://seer.ufrgs.br/Movimento/article/view/113041. Acesso em: [dia] [mês abreviado]. [ano]. DOI: https://doi.org/10.22456/1982$\underline{8918.113041}$

\section{RESPONSABILIDADE EDITORIAL}

Alex Branco Fraga*, Elisandro Schultz Wittizorecki*, Ivone Job*, Mauro Myskiw*, Raquel da Silveira*

*Universidade Federal do Rio Grande do Sul, Escola de Educação Física, Fisioterapia e Dança, Porto Alegre, RS, Brasil. 